\title{
Impact of non-separable incidence rates on global dynamics of virus model with cell-mediated, humoral immune responses
}

\author{
Yoichi Enatsu ${ }^{a, *}$, Jinliang Wang ${ }^{b}$, Toshikazu Kuniya ${ }^{c}$ \\ ${ }^{a}$ Department of Applied Mathematics, Tokyo University of Science, 1-3 Kagurazaka, Shinjuku-ku, Tokyo 162-8601, Japan. \\ ${ }^{b}$ School of Mathematical Science, Heilongjiang University, Harbin 150080, China. \\ ${ }^{c}$ Graduate School of System Informatics, Kobe University, 1-1 Rokkodai-cho, Nada-ku, Kobe 657-8501, Japan.
}

Communicated by A. Atangana

\begin{abstract}
In this paper, we study the dynamical behavior of a virus model into which cell-mediated and humoral immune responses are incorporated. The global stability of an infection-free equilibrium and four infected equilibria is established via a Lyapunov functional approach. The present construction methods are applicable to a wide range of incidence rates that are monotone increasing with respect to concentration of uninfected cells and concave with respect to the concentration of free virus particles. In addition, when the incidence rate is monotone increasing with respect to concentration of free virus particles, the functional approach plays an important role in determining the global stability of each of the four infected equilibria. This implies that the dynamical behavior of virus prevalence would be determined by basic reproduction numbers when the "saturation effect" for free virus particles appears. We point out that the incidence rate includes not only separable incidence rate but also non-separable incidence rate such as standard incidence and Beddington-DeAngelis functional response. (C)2017 All rights reserved.
\end{abstract}

Keywords: Virus infection model, delay, global stability, incidence rate.

2010 MSC: 34K20, 34K25, 92D30.

\section{Introduction}

Modeling the dynamics of virus infection has been extensively developed in order to investigate shortor long-term progression of viral production. The pioneering work is done by Nowak and Bangham [16], in which the relation between antiviral immune responses, virus load, and virus diversity are theoretically understood by formulating systems with ordinary differential equations describing the population dynamics of immune responses to virus load. Motivated by their work, many studies have subsequently focused on the global stability of equilibria in models for viral dynamics which play a crucial role in clarifying and evaluating treatment strategies for infections and establishing thresholds for treatment rates $[11,12,14,15,20,24,29]$. In this paper, we focus our attention on the global stability of steady states for these models, because this should enhance our understanding of virus dynamics, which gives us a

\footnotetext{
*Corresponding author

Email addresses: yenatsu@rs.tus.ac.jp (Yoichi Enatsu), jinliangwang@aliyun.com (Jinliang Wang), tkuniya@port.kobe-u.ac.jp (Toshikazu Kuniya)

doi:10.22436/jnsa.010.10.07
} 
detailed information and various insights on whether a disease will die out or not, and the mechanisms of specific immune responses. A typical recent model of viral dynamics containing humoral immune responses is given by Wang et al. [22] as follows:

$$
\left\{\begin{array}{l}
x^{\prime}(t)=\lambda-d x(t)-f(x(t), v(t)) v(t), \\
y^{\prime}(t)=e^{-m_{1} \tau_{1}} f\left(x\left(t-\tau_{1}\right), v\left(t-\tau_{1}\right)\right) v\left(t-\tau_{1}\right)-\delta y(t), \\
v^{\prime}(t)=k e^{-m_{2} \tau_{2}} y\left(t-\tau_{2}\right)-c v(t)-q a(t) v(t), \\
a^{\prime}(t)=g a(t) v(t)-b a(t)
\end{array}\right.
$$

The variables $x, y$ and $v$ denote the concentrations of uninfected cells, infected cells and free virus particles, respectively. The variable a denotes the concentration of B cells, whose principal function is to make antibodies against soluble antigens. The nonnegative constant $\tau_{1}$, referred to as an intracellular delay, denotes the time taken for the production of new virus particles after a virus has entered a cell. The nonnegative constant $\tau_{2}$ denotes the time taken for the maturation of newly produced viruses (see Ouifki et al. [17]). All other parameters are positive constants. The model (1.1) with $f(x, v)=k x$ (i.e., a bilinear incidence $f(x, v) v=k x v)$ is equivalent to that presented by Wang et al. [27, Section 3].

For the non-separable function $f \in C^{1}\left(\mathbb{R}_{+}^{2}, \mathbb{R}\right)$, denoting the average number of infected cells, let us set up the hypotheses in Wang et al. [22, H1-H4]:

$\left(A_{1}\right) \quad f(x, v) \geqslant 0$ and $f(x, v)=0$ if and only if $x=0$.

$\left(\mathrm{A}_{2}\right) \frac{\partial \mathrm{f}(\mathrm{x}, v)}{\partial x}>0$ for all $x \geqslant 0$ and $v \geqslant 0$.

$\left(\mathrm{A}_{3}\right) \frac{\partial f(x, v)}{\partial v} \leqslant 0$ for all $x \geqslant 0$ and $v \geqslant 0$.

$\left(\mathrm{A}_{4}\right) \frac{\partial(\mathrm{f}(\mathrm{x}, v) v)}{\partial v}>0$ for all $x>0$ and $v \geqslant 0$.

The hypothesis $\left(\mathrm{A}_{1}\right)$ biologically indicates that the per-capita number of newly virus-infected cells is always nonnegative. The hypothesis $\left(\mathrm{A}_{2}\right)$ indicates that the more the amount of uninfected cells is, then the more the per-capita number of newly virus-infected cells will be, for a fixed number of free virus particles. The hypothesis $\left(A_{3}\right)$ indicates that the more the amount of virus is, then the less the per-capita number of newly virus-infected cells will be for a fixed number of uninfected cells. The hypothesis $\left(\mathrm{A}_{4}\right)$ indicates that the more the amount of virus is, then the more the number of cells that are newly infected will be. All of the above hypotheses include the following incidence rates.

$$
\begin{gathered}
\text { Separable }\left\{\begin{array}{c}
\text { (i) } \quad \text { Bilinear incidence rate }([16,19]) \quad f(x, v) v=x v, \\
\text { (ii) Saturated incidence rate }([18]) \quad f(x, v) v=\frac{x v}{1+\alpha v}, \alpha>0,
\end{array}\right. \\
\text { Non-separable }\left\{\begin{array}{c}
\text { (iii) } \begin{array}{c}
\text { Standard incidence rate }([10]) \\
f(x, v) v=\frac{x v}{x+v}
\end{array} \\
\text { (iv) Beddington-DeAngelis functional response }([2,5]) \\
f(x, v) v=\frac{x v}{1+\alpha_{1} x+\alpha_{2} v}, \alpha_{1}, \alpha_{2}>0 .
\end{array}\right.
\end{gathered}
$$

In addition to the effect of humoral immune responses, some authors investigated the effect of cellmediated cytotoxic T lymphocytes (CTLs) immune response on the dynamics of cell infection (see, e.g., [1, $3,6,7]$ and the references therein). In contrast to the models with humoral immune response, it is asserted that these models including CTL response displays rich dynamics when the delays are incorporated; the global stability of the equilibria is completely determined by threshold parameters [11, 15, 24, 29], whereas 
periodic solutions arise through the Hopf bifurcations $[4,25,26,30]$. The issues investigated in these models were related to understanding whether the viruses can be cleared, whether life long immunity can be achieved in the host, and whether sustained oscillatory viral loads can be observed. Later, Yan and Wang [28] formulated the following model incorporating both cell-mediated and antibody responses with delays:

$$
\left\{\begin{array}{l}
x^{\prime}(t)=\lambda-d x(t)-k x(t) v(t), \\
y^{\prime}(t)=k x(t-\tau) v(t-\tau) e^{-s \tau}-\delta y(t)-p y(t) z(t), \\
v^{\prime}(t)=\delta N y(t)-c v(t)-q a(t) v(t), \\
z^{\prime}(t)=\beta y(t) z(t)-\gamma z(t) \\
a^{\prime}(t)=g a(t) v(t)-b a(t)
\end{array}\right.
$$

Here the variables $x, y, v, z$ and a denote the concentrations of uninfected cells, infected cells, free virus particles, CTL responses and antibody responses, respectively. On the other hand, in the literature of epidemiology, it has increasingly been asserted that the incidence rate, characterizing the rate of newly infected cells, should be written by not bilinear but general nonlinear functions (see, e.g., [8, 9, 26]).

We point out that since both the model (1.1) does not consider cell-mediated cytotoxic T lymphocytes (CTLs) immune response and model (1.2) does not incorporate non-separable incidence rate, in this paper, we combine these two considerations to formulate our model. We aim to establish global stability results and identify sufficient conditions under which oscillations are impossible for a viral model with humoral immune responses, CTL immune response, and a non-separable incidence rate. For complete characterization of the global dynamics, we define some basic reproduction numbers which serve as threshold parameters that predict whether an infection will go to extinction or persist. The global stability scenario of infection equilibrium is achieved by LaSalle invariant principle and the Lyapunov functional approach.

This paper is organized as follows. In Section 2, we introduce our model by incorporating a class of non-separable incidence rates. The model is governed by a system of delay differential equations. We also specify the basic reproduction numbers of the model and focus on the existence of four infection equilibria. In Section 3, we establish the global attractivity and (uniform) stability of each of the four equilibria by constructing Lyapunov functionals based on LaSalle invariance principle. In Section 4, we offer some concluding remarks on our stability results.

\section{The model and preliminaries}

Let us consider the following model:

$$
\left\{\begin{aligned}
x^{\prime}(t) & =\lambda-d x(t)-f(x(t), v(t)) v(t) \\
y^{\prime}(t) & =\int_{0}^{\infty} G_{1}(\tau) f(x(t-\tau), v(t-\tau)) v(t-\tau) d \tau-\delta y(t)-p y(t) z(t), \\
v^{\prime}(t) & =\delta N \int_{0}^{\infty} G_{2}(\tau) y(t-\tau) d \tau-c v(t)-q a(t) v(t) \\
z^{\prime}(t) & =\beta y(t) z(t)-\gamma z(t) \\
a^{\prime}(t) & =g a(t) v(t)-b a(t)
\end{aligned}\right.
$$

The uninfected cells are produced at a constant rate $\lambda$ and die at a per capita rate $d$. The infected cells are assumed to die at a rate $\delta$ due to the action of virus, each releasing $N$ new virus particles as they are lysed. Virus particles are cleared from the system at a rate $c$. The infected cells are also killed via mass action kinetics by CTLs, which is described by pyz. Virus particles are also neutralized via mass action kinetics by antibodies, which is described by qav. CTLs are produced at a rate proportional to the abundances of CTLs and infected cells, $\beta y z$, and die at a per capita rate $\gamma$. The antibody responses are activated at a rate proportional to the abundances of antibodies and free viruses, gav, and die at a per capita rate $b$. To account for the time lag between viral entry into a target cell and the production of new virus particles, two distributed intracellular delays are introduced with kernel functions given by $G_{i}(\tau)=f_{i}(\tau) e^{-m_{i} \tau}$, 
$i=1,2 . G_{1}(\tau)$ is the probability that target cells contacted by the virus particles at time $t-\tau$ survived $\tau$ time units and become infected at time $t$ and $G_{2}(\tau)$ is the probability that a cell infected at time $t-\tau$ starts to yield new infectious virus at time $t$. All the parameters are positive constants. The function $f$ is assumed to satisfy the hypotheses $\left(\mathrm{A}_{1}\right)-\left(\mathrm{A}_{4}\right)$. We also note that the model is equivalent to the model studied by Wang et al. [24] if the incidence rate $f(x, v) v$ is of the separable form $x F(v)$.

The following assumption:

$\left(A_{5}\right) G_{i}(\tau)>0$, for $\tau>0$, and $0<a_{i}:=\int_{0}^{\infty} G_{i}(\xi) d \xi \leqslant 1, i=1,2 ;$

is also used widely in the literatures when describing delay kernels. For convenience, we rewrite (2.1) as

$$
\left\{\begin{array}{l}
x^{\prime}(t)=\lambda-d x(t)-f(x(t), v(t)) v(t) \\
y^{\prime}(t)=\alpha_{1} \int_{0}^{\infty} g_{1}(\xi) f(x(t-\xi), v(t-\xi)) v(t-\xi) d \xi-\delta y(t)-p y(t) z(t), \\
v^{\prime}(t)=\alpha_{2} \int_{0}^{\infty} g_{2}(\xi) y(t-\xi) d \xi-c v(t)-q a(t) v(t) \\
z^{\prime}(t)=\beta y(t) z(t)-\gamma z(t) \\
a^{\prime}(t)=g a(t) v(t)-b a(t)
\end{array}\right.
$$

where $\alpha_{1}=a_{1}, \alpha_{2}=N \delta a_{2}$ and $g_{i}(\xi)=\frac{G_{i}(\xi)}{a_{i}}$ for $i=1,2$. We recall that $a_{i}=\int_{0}^{\infty} G_{i}(\xi) d \xi$, thus $\int_{0}^{\infty} g_{i}(\xi) d \xi=1$.

We consider a suitable phase and a feasible region. Nonnegative initial functions are given as follows:

$$
(x(\theta), y(\theta), v(\theta), z(\theta), a(\theta))=\left(\phi_{1}(\theta), \phi_{2}(\theta), \phi_{3}(\theta), \phi_{4}(\theta), \phi_{5}(\theta)\right)=\phi(\theta) \in \mathrm{UC}_{\psi}\left((-\infty, 0], \mathbb{R}_{+}^{5}\right),
$$

where $\mathbb{R}_{+}^{5}=\left\{\left(x_{1}, x_{2}, x_{3}, x_{4}, x_{5}\right) \in \mathbb{R}^{5}: x_{i} \geqslant 0, i=1,2,3,4,5\right\}$ and

$$
\begin{aligned}
\mathrm{UC}_{\psi}\left((-\infty, 0], \mathbb{R}_{+}^{5}\right):=\{ & \phi \in \mathrm{C}\left((-\infty, 0], \mathbb{R}_{+}^{5}\right):\|\phi\|_{\psi}=\sup _{\mathrm{s} \leqslant 0} \frac{|\phi(s)|}{\psi(s)}<\infty, \\
& \left.\frac{\phi(s)}{\psi(s)} \text { is uniformly continuous on }(-\infty, 0]\right\} .
\end{aligned}
$$

Here we assume that $\psi:(-\infty, 0] \longrightarrow[1, \infty)$ satisfies the following properties:

(1) $\psi$ is continuous and nonincreasing on $(-\infty, 0]$ with $\psi(0)=1$;

(2) $\frac{\psi(s+\mathfrak{u})}{\psi(s)} \rightarrow 1$ uniformly on $(-\infty, 0]$ as $\mathfrak{u} \rightarrow-0$;

(3) $\psi(s) \rightarrow \infty$ as $s \rightarrow-\infty$.

We note that $\mathrm{UC}_{\psi}$ is a Banach space with norm $\|\cdot\|_{\psi}$. Moreover, if the function $\psi$ satisfies assumptions (1)-(3), then $\mathrm{UC}_{\psi}$ is an admissible Banach space. Thus, for system (2.2), existence results of the Peano type hold (see, for details, Kuang [13, Corollary 5.2]).

It follows from the fundamental theory for integral-differential equations that there exists a $T_{\phi}>0$ such that system (2.2) with (2.3) has a unique solution on the interval $\left[0, T_{\phi}\right)$. The following theorem shows that for positive initial values, the solution remains positive and is bounded, implying $\mathrm{T}_{\phi}=\infty$, that is, the solution exists globally in time. The proof is omitted because it is quite similar to that in Wang et al. [24, Theorem 2.1].

Theorem 2.1. Let $(x(t), y(t), v(t), z(t), a(t))^{\top}$ be the unique solution to system (2.2) with (2.3). Then $x(t), y(t)$, $v(t), z(t)$ and $a(t)$ are nonnegative for all $t>0$. Moreover, all solutions $(x(t), y(t), v(t), z(t), a(t))^{\top}$ of system (2.2) with $x(t)>0, y(t)>0, v(t)>0, z(t)>0$ and $\mathrm{a}(\mathrm{t})>0$ are ultimately bounded.

From Theorem 2.1, we can easily verify that $\omega$-limit sets of system (2.2) are contained in the following bounded feasible region:

$$
\Gamma=\left\{(x, y, v, z, a) \in \mathbb{R}_{+}^{5}:|x| \leqslant \frac{\lambda}{d},|y|,|z| \leqslant \frac{\lambda a_{1}}{\min \{d, \delta, \gamma\}},|v|,|a| \leqslant \frac{\delta a_{2} N_{\frac{1}{\min \{d, \delta, \gamma\}}}}{\min \{c, b\}}\right\} .
$$

We can easily verify that the region $\Gamma$ is positively invariant with respect to system (2.2). 
2.1. Reproduction numbers and existence of positive equilibria

The equilibria of system (2.2) satisfy the following equalities:

$$
\left\{\begin{array}{l}
\lambda-d x-f(x, v) v=0 \\
\alpha_{1} f(x, v) v-\delta y-p y z=0 \\
\alpha_{2} y-c v-q a v=0 \\
\beta y z-\gamma z=0 \\
g a v-b a=0
\end{array}\right.
$$

System (2.4) always has an infection-free equilibrium $E_{0}=(\lambda / d, 0,0,0,0)$ if $v=0, y=0, z=0$ and $a=0$. By simple calculation, we see that an immune-free equilibrium $E_{1}=\left(x_{1}, y_{1}, v_{1}, 0,0\right)$ exists if and only if a positive root of the equation $F_{1}(x)=0$ on $(0, \lambda / d)$, where

$$
F_{1}(x)=f\left(x, \frac{\alpha_{1} \alpha_{2}}{\delta c}\right)-\frac{\delta c}{\alpha_{1} \alpha_{2}} .
$$

The basic reproduction number is one of the most important concepts in viral infection models which serves as a threshold parameter that predicts whether the infection will go to extinction or persist. We define the basic reproduction number for viral infection as

$$
\mathfrak{R}_{0}:=\frac{\alpha_{1} \alpha_{2}}{\mathrm{c} \delta} \mathrm{f}\left(\mathrm{x}_{0}, 0\right) .
$$

From assumptions $\left(A_{1}\right)$ and $\left(A_{2}\right)$, the function $F_{1}(x)$ is strictly monotonically increasing with respect to $x$. It follows that

$$
F_{1}(0)=-\frac{\delta c}{\alpha_{1} \alpha_{2}}<0, \text { and } F_{1}\left(\frac{\lambda}{d}\right)=f\left(x_{0}, 0\right)-\frac{\delta c}{\alpha_{1} \alpha_{2}}=\frac{\alpha_{1} \alpha_{2}}{\delta c}\left(\Re_{0}-1\right)
$$

There exists a unique $x_{1} \in(0, \lambda / d)$ such that $F_{1}\left(x_{1}\right)=0$ if and only if $\mathfrak{R}_{0}>1$. By the relation

$$
y_{1}=\frac{\alpha_{1}(\lambda-d x)}{\delta} \text { and } v_{1}=\frac{\alpha_{1} \alpha_{2}(\lambda-d x)}{\delta c}
$$

we get an immune-free equilibrium $E_{1}=\left(x_{1}, y_{1}, v_{1}, 0,0\right)$.

Next we consider $E_{2}=\left(x_{2}, y_{2}, v_{2}, z_{2}, 0\right)$. For $z \neq 0$ and $a=0$, from the fourth equation of (2.4), we can get $y_{2}=\frac{\gamma}{\beta}<y_{1}$, which is equivalent to

$$
\mathfrak{R}_{1}:=\frac{\beta y_{1}}{\gamma}>1
$$

Then the first equation of (2.4) becomes

$$
f\left(x, \frac{\alpha_{2} \gamma}{\beta c}\right) \frac{\alpha_{2} \gamma}{\beta c}-\lambda+d x=0,
$$

and we have

$$
y_{2}=\frac{\gamma}{\beta}, v_{2}=\frac{\alpha_{2} \gamma}{\beta c} \text { and } z_{2}=\frac{\beta \alpha_{1}\left(\lambda-\mathrm{d} x_{2}\right)}{p \gamma}-\frac{\delta}{p} .
$$

Since $z_{2}>0$ we have $x_{2}<x^{*}$, where

$$
x^{*}=\frac{\lambda}{d}-\frac{\delta \gamma}{d \beta \alpha_{1}} .
$$

It follows that the existence of equilibrium requires $x^{*}>0$ and (2.7) has a unique positive root $x=x_{2} \in$ $\left(0, x^{*}\right)$. We denote

$$
F_{2}(x)=f\left(x, \frac{\alpha_{2} \gamma}{\beta c}\right) \frac{\alpha_{2} \gamma}{\beta c}-\lambda+d x
$$


We know $F_{2}(x)$ is strictly monotonically increasing with respect to $x$ from hypothesis $\left(A_{2}\right)$. It is clear that $F_{2}(0)=-\lambda<0$ holds and

$$
\begin{aligned}
F_{2}\left(x^{*}\right) & =f\left(x^{*}, \frac{\alpha_{2} \gamma}{\beta c}\right) \frac{\alpha_{2} \gamma}{\beta c}-\lambda+d x^{*} \\
& =f\left(x^{*}, \frac{\alpha_{2} \gamma}{\beta c}\right) \frac{\alpha_{2} \gamma}{\beta c}-\frac{\delta \gamma}{\beta \alpha_{1}} \\
& =\frac{\delta \gamma}{\beta \alpha_{1}}\left(f\left(x^{*}, \frac{\alpha_{2} \gamma}{\beta c}\right) \frac{\alpha_{1} \alpha_{2}}{\delta c}-1\right) .
\end{aligned}
$$

Therefore, there exists a unique real value $x_{2} \in\left(0, x^{*}\right)$ such that $F_{2}\left(x_{2}\right)=0$ if and only if $\frac{\lambda d \beta \alpha_{1}}{d \delta \gamma}>1$ and $f\left(x^{*}, \frac{\alpha_{2} \gamma}{\beta c}\right) \frac{\alpha_{1} \alpha_{2}}{\delta c}>1$.

Let us remark that the assumptions $\left(\mathrm{A}_{1}\right)$ and $\left(\mathrm{A}_{2}\right)$ imply

$$
f\left(x^{*}, \frac{\alpha_{2} \gamma}{\beta c}\right)<f\left(\frac{\lambda}{d}, \frac{\alpha_{2} \gamma}{\beta c}\right)<f\left(\frac{\lambda}{d}, 0\right),
$$

so that, we get the unique equilibrium $E_{2}=\left(x_{2}, y_{2}, v_{2}, z_{2}, 0\right)$ if and only if $\Re_{1}>1$. Here, $\Re_{1}$ denotes the average number of the CTL immune cells activated by infected cells when virus infection is successful and humoral immune responses have not been established. Note that $y_{1}$ is the number of infected cells at $E_{1}$ and $1 / r$ is the average life-span of CTL cells.

Next, we consider $E_{3}=\left(x_{3}, y_{3}, v_{3}, 0, a_{3}\right)$. For $a \neq 0$ and $z=0$, from the fifth equation of (2.4), we can get $v_{3}=\frac{\mathrm{b}}{\mathrm{g}}<v_{1}$, which is equivalent to

$$
\mathfrak{R}_{2}:=\frac{\mathrm{g} v_{1}}{\mathrm{~b}}>1
$$

Then the first equation of (2.4) becomes

$$
k x f\left(\frac{b}{g}\right)-\lambda+d x=0
$$

and we have

$$
y_{3}=\frac{a_{1}\left(\lambda-d x_{3}\right)}{\delta} \text { and } a_{3}=\frac{N a_{1} a_{2} g\left(\lambda-d x_{3}\right)}{q b}-\frac{c}{q} .
$$

It follows that the equation (2.9) has a unique positive root $x=x_{3} \in(0, \lambda / d)$. Therefore we get the unique equilibrium $E_{3}=\left(x_{3}, y_{3}, v_{3}, 0, a_{3}\right)$ if and only if $\mathfrak{R}_{2}>1$. Here, $\mathfrak{R}_{2}$ denotes the average number of humoral immune cells activated by virus when virus infection is successful and CTL responses have not been established. Note that $v_{1}$ is the number of free viruses at $E_{1}$ and $1 / b$ is the average life-span of antibody cells.

Finally we consider $E_{4}=\left(x_{4}, y_{4}, v_{4}, z_{4}, a_{4}\right)$. For $a \neq 0$ and $z \neq 0$, from the fourth and fifth equations of (2.4), we can get

$$
y_{4}=\frac{\gamma}{\beta} \text { and } v_{4}=\frac{b}{g} .
$$

From the second equation of (2.4), we can get

$$
z=\frac{\delta}{p}\left(\frac{\beta k a_{1} \times f\left(\frac{b}{g}\right)}{\gamma \delta}-1\right)
$$

Note that $\frac{k a_{1} \times f\left(\frac{b}{g}\right)}{\delta}=y_{3}=\frac{a_{1}(\lambda-d x)}{\delta}$ is the number of infected cells at $E_{3}$. We define the CTL immune competitive reproductive number $\mathfrak{R}_{3}$ for system (2.2) as

$$
\mathfrak{R}_{3}:=\frac{\beta y_{3}}{\gamma},
$$


where $1 / \gamma$ is the average life-span of CTL cells. Here, $\mathfrak{R}_{3}$ denotes the average number of the CTL immune cells activated by infected cells under the condition that humoral immune responses have been established.

From the third equation of (2.4), we can get

$$
a=\frac{c}{q}\left(\frac{g \delta \mathrm{Na}_{2} \gamma}{\beta b c}-1\right)
$$

Note that $\frac{N \delta a_{2} \gamma}{\beta c}$ is the number of the viruses at $E_{2}$. Here the humoral immune competitive reproductive number $\mathfrak{R}_{4}$ for system (2.2) is denoted as follows.

$$
\mathfrak{R}_{4}:=\frac{\mathrm{g} v_{2}}{\mathrm{~b}} .
$$

Note that $1 / \mathrm{b}$ is the average life-span of antibody cells and thus, $\mathfrak{R}_{4}$ denotes the average number of the humoral immune cells activated by viruses under the condition that CTL immune responses have been established.

When $\mathfrak{R}_{3}>1$ and $\mathfrak{R}_{4}>1$, CTL and humoral immune responses can be established simultaneously, and there exists an interior equilibrium $E_{4}=\left(x_{4}, y_{4}, v_{4}, z_{4}, a_{4}\right)$.

Now we are in a position to state the following theorem on the existence of equilibria under the basic reproduction numbers.

Theorem 2.2. Let $\mathfrak{R}_{0}, \mathfrak{R}_{1}, \mathfrak{R}_{2}, \mathfrak{R}_{3}$ and $\mathfrak{R}_{4}$ be defined by (2.5), (2.6), (2.8), (2.10) and (2.11), respectively.

(i) System (2.2) always has an infection-free equilibrium $\mathrm{E}_{0}$.

(ii) System (2.2) has an immune-free infection equilibrium $\mathrm{E}_{1}$ when $\mathfrak{R}_{0}>1$.

(iii) System (2.2) has an infection equilibrium $\mathrm{E}_{2}$ with only CTL immune responses when $\mathfrak{R}_{1}>1$.

(iv) System (2.2) has an infection equilibrium $\mathrm{E}_{3}$ with only humoral immune responses when $\mathfrak{R}_{2}>1$.

(v) System (2.2) has an infection equilibrium $\mathrm{E}_{4}$ with both CTL responses and humoral immune responses when $\mathfrak{R}_{3}>1$ and $\mathfrak{R}_{4}>1$.

\section{Global dynamics}

We define

$$
H_{i}(t):=\int_{t}^{\infty} g_{i}(\xi) d \xi, \quad i=1,2
$$

From the definition of the function $g_{1}$ with the assumption $\left(A_{5}\right)$, we note that $H_{1}(0)=1, H_{1}(\infty)=0$ and $\frac{\mathrm{d} \mathrm{H}_{1}(\mathrm{t})}{\mathrm{dt}}=-\mathrm{g}_{1}(\mathrm{t})$ holds.

The following theorem indicates that the viruses are eventually cleared.

Theorem 3.1. When $\mathfrak{R}_{0} \leqslant 1$, the infection-free equilibrium $\mathrm{E}_{0}$ is globally asymptotically stable.

Proof. Define the following Lyapunov functional:

$$
\begin{aligned}
\mathrm{V}_{1}(\mathrm{t})= & \mathrm{u}_{x_{0}}(\mathrm{t})+\frac{1}{\alpha_{1}} \mathrm{y}(\mathrm{t})+\frac{\delta}{\alpha_{1} \alpha_{2}} v(\mathrm{t})+\frac{p}{\alpha_{1} \beta} z(t)+\frac{\delta q}{\alpha_{1} \alpha_{2} g} a(t) \\
& +\int_{0}^{\infty} \mathrm{H}_{1}(\xi) f(x(t-\xi), v(t-\xi)) v(t-\xi) d \xi+\frac{\delta}{\alpha_{1}} \int_{0}^{\infty} H_{2}(\zeta) y(t-\xi) d \xi
\end{aligned}
$$

where

$$
u_{x_{0}}(t)=x(t)-x_{0}-\int_{x_{0}}^{x(t)} \frac{f\left(x_{0}, 0\right)}{f(s, 0)} d s
$$


We calculate the time derivative of $V_{1}$ as follows.

$$
\begin{aligned}
V_{1}^{\prime}(t)= & \left(1-\frac{f\left(x_{0}, 0\right)}{f(x(t), 0)}\right)(\lambda-d x(t)-f(x(t), v(t)) v(t)) \\
& +\frac{1}{\alpha_{1}}\left(\alpha_{1} \int_{0}^{\infty} g_{1}(\xi) f(x(t-\xi), v(t-\xi)) v(t-\xi) d \xi-\delta y(t)-p y(t) z(t)\right) \\
& +\frac{\delta}{\alpha_{1} \alpha_{2}}\left(\alpha_{2} \int_{0}^{\infty} g_{2}(\xi) y(t-\xi) d \xi-c v(t)-q a(t) v(t)\right) \\
& +\frac{p}{\alpha_{1} \beta}(\beta y(t) z(t)-\gamma z(t))+\frac{\delta q}{\alpha_{1} \alpha_{2} g}(g a(t) v(t)-b a(t)) \\
& +f(x(t), v(t)) v(t)-\int_{0}^{\infty} g_{1}(\xi) f(x(t-\xi), v(t-\xi)) v(t-\xi) d \xi+\frac{\delta}{\alpha_{1}} y(t)-\frac{\delta}{\alpha_{1}} \int_{0}^{\infty} g_{2}(\xi) y(t-\xi) d \xi \\
= & d\left(1-\frac{f\left(x_{0}, 0\right)}{f(x(t), 0)}\right)\left(x_{0}-x(t)\right)+\frac{f\left(x_{0}, 0\right)}{f(x(t), 0)} f(x(t), v(t)) v(t)-\frac{p}{\alpha_{1}} y(t) z(t)-\frac{\delta c}{\alpha_{1} \alpha_{2}} v(t)-\frac{\delta q}{\alpha_{1} \alpha_{2}} a(t) v(t) \\
& +\frac{p}{\alpha_{1}} y(t) z(t)-\frac{p \gamma}{\alpha_{1} \beta} z(t)+\frac{\delta q}{\alpha_{1} \alpha_{2}} a(t) v(t)-\frac{\delta q b}{\alpha_{1} \alpha_{2} g} a(t) \\
= & d\left(1-\frac{f\left(x_{0}, 0\right)}{f(x(t), 0)}\right)\left(x_{0}-x(t)\right)-\frac{p \gamma}{\alpha_{1} \beta} z(t)-\frac{\delta q b}{\alpha_{1} \alpha_{2} g} a(t)+\frac{\delta c}{\alpha_{1} \alpha_{2}}\left(\Re_{0} \frac{f(x(t), v(t))}{f(x(t), 0)}-1\right) v(t) .
\end{aligned}
$$

From the assumption $\left(A_{2}\right)$, since $f(x, v)$ is strictly monotonically increasing with respect to $x$, we have

$$
d\left(1-\frac{f\left(x_{0}, 0\right)}{f(x(t), 0)}\right)\left(x_{0}-x(t)\right) \leqslant 0 .
$$

It follows from $\left(A_{3}\right)$ that $f(x, v)$ is monotonically decreasing with respect to $v$, which implies in addition to $\mathfrak{R}_{0} \leqslant 1$ that

$$
\mathfrak{R}_{0} \frac{f(x(t), v(t))}{f(x(t), 0)}-1 \leqslant 0 .
$$

Consequently, one can see that $V_{1}^{\prime}(t) \leqslant 0$, and $V_{1}^{\prime}(t)=0$ if $x(t)=x_{0}, z(t)=0$ and $a(t)=0$ for $\mathfrak{R}_{0} \leqslant 1$. Hence, every solution of (2.2) tends to $M_{0}$, where $M_{0}$ is the largest invariant subset of

$$
\left\{(x, y, v, z, a) \in \Gamma: V_{1}^{\prime}=0\right\} .
$$

It can be easily verified that $M_{0}$ is singleton $\left\{E_{0}\right\}$ because every element of $M_{0}$ satisfies $y(t)=0, v(t)=0$ for all $t>0$. From the LaSalle invariance principle (see, e.g., Kuang [13, Corollary 5.2]), $E_{0}$ is globally attractive. In addition, since $V_{1}$ is estimated by a positive definite function from below

$$
v_{1}(t) \geqslant u_{x_{0}}(t)+\frac{1}{\alpha_{1}} y(t)+\frac{\delta}{\alpha_{1} \alpha_{2}} v(t)+\frac{p}{\alpha_{1} \beta} z(t)+\frac{\delta q}{\alpha_{1} \alpha_{2} g} a(t)
$$

$E_{0}$ is uniformly stable. This implies that $E_{0}$ is globally asymptotically stable.

The following theorem indicates that the infection becomes chronic but with no persistent CTL immune response and antibody immune response.

Theorem 3.2. When $\mathfrak{R}_{0}>1, \mathfrak{R}_{1} \leqslant 1$ and $\mathfrak{R}_{2} \leqslant 1$, the immune-free infection equilibrium $\mathrm{E}_{1}$ is globally asymptotically stable.

Proof. We will make use of the equations for the elements of $E_{1}$ in order to simplify the expressions:

$$
\left\{\begin{array}{l}
\lambda=d x_{1}+f\left(x_{1}, v_{1}\right) v_{1} \\
\alpha_{1} f\left(x_{1}, v_{1}\right) v_{1}=\delta y_{1} \\
\alpha_{2} y_{1}=c v_{1}
\end{array}\right.
$$


Let us define $g(\mu)=\mu-1-\ln \mu$. One can see that the function $g$ is positive definite: $g(\mu) \geqslant 0$ for $\mu>0$ and $g(\mu)=0$ if and only if $\mu=1$. The function $g$ is called the Volterra-type function which here plays a crucial role in constructing suitable Lyapunov functionals below. Define the following Lyapunov functional:

$$
v_{2}(t)=u_{x_{1}}(t)+\frac{1}{\alpha_{1}} y_{1} g\left(\frac{y(t)}{y_{1}}\right)+\frac{\delta}{\alpha_{1} \alpha_{2}} v_{1} g\left(\frac{v(t)}{v_{1}}\right)+\frac{p}{\alpha_{1} \beta} z(t)+\frac{\delta q}{\alpha_{1} \alpha_{2} g} a(t)+W_{1}(t)+W_{2}(t),
$$

where

$$
\begin{aligned}
& \mathrm{u}_{x_{1}}(\mathrm{t})=x(\mathrm{t})-\mathrm{x}_{1}-\int_{\mathrm{x}_{1}}^{\mathrm{x}(\mathrm{t})} \frac{f\left(\mathrm{x}_{1}, v_{1}\right)}{\mathrm{f}\left(\mathrm{s}, v_{1}\right)} d s, \\
& \mathrm{~W}_{1}(\mathrm{t})=\mathrm{f}\left(\mathrm{x}_{1}, v_{1}\right) v_{1} \int_{0}^{\infty} \mathrm{H}_{1}(\xi) g\left(\frac{f(x(t-\xi), v(t-\xi)) v(t-\xi)}{f\left(x_{1}, v_{1}\right) v_{1}}\right) d \xi, \\
& W_{2}(t)=\frac{\delta}{\alpha_{1}} y_{1} \int_{0}^{\infty} H_{2}(\xi) g\left(\frac{y(t-\xi)}{y_{1}}\right) d \xi .
\end{aligned}
$$

We then obtain

$$
\begin{aligned}
W_{1}^{\prime}(t)= & f\left(x_{1}, v_{1}\right) v_{1} \int_{0}^{\infty} H_{1}(\xi) \frac{d g\left(\frac{f(x(t-\xi), v(t-\xi)) v(t-\xi)}{f\left(x_{1}, v_{1}\right) v_{1}}\right)}{d t} d \xi \\
= & -f\left(x_{1}, v_{1}\right) v_{1} \int_{0}^{\infty} H_{1}(\xi) \frac{d g\left(\frac{f(x(t-\xi), v(t-\xi)) v(t-\xi)}{f\left(x_{1}, v_{1}\right) v_{1}}\right)}{d \xi} d \xi \\
= & f(x(t), v(t)) v(t)-\int_{0}^{\infty} g_{1}(\xi) f(x(t-\xi), v(t-\xi)) v(t-\xi) d \xi \\
& +f\left(x_{1}, v_{1}\right) v_{1} \int_{0}^{\infty} g_{1}(\xi) \ln \frac{f(x(t-\xi), v(t-\xi)) v(t-\xi)}{f(x(t), v(t)) v(t)} d \xi \\
W_{2}^{\prime}(t)= & \frac{\delta}{\alpha_{1}} y(t)-\frac{\delta}{\alpha_{1}} \int_{0}^{\infty} g_{2}(\xi) y(t-\xi) d \xi+\frac{\delta}{\alpha_{1}} y_{1} \int_{0}^{\infty} g_{2}(\xi) \ln \frac{y(t-\xi)}{y(t)} d \xi
\end{aligned}
$$

Calculating the time derivative of $V_{2}(t)$ gives

$$
\begin{aligned}
V_{2}^{\prime}(t)= & \left(1-\frac{f\left(x_{1}, v_{1}\right)}{f\left(x(t), v_{1}\right)}\right)(\lambda-d x(t)-f(x(t), v(t)) v(t)) \\
& +\frac{1}{\alpha_{1}}\left(1-\frac{y_{1}}{y(t)}\right)\left(\alpha_{1} \int_{0}^{\infty} g_{1}(\xi) f(x(t-\xi), v(t-\xi)) v(t-\xi) d \xi-\delta y(t)-p y(t) z(t)\right) \\
& +\frac{\delta}{\alpha_{1} \alpha_{2}}\left(1-\frac{v_{1}}{v(t)}\right)\left(\alpha_{2} \int_{0}^{\infty} g_{2}(\xi) y(t-\xi) d \xi-c v(t)-q a(t) v(t)\right) \\
& +\frac{p}{\alpha_{1} \beta}(\beta y(t) z(t)-\gamma z(t))+\frac{\delta q}{\alpha_{1} \alpha_{2} g}(g a(t) v(t)-b a(t)) \\
& +f(x(t), v(t)) v(t)-\int_{0}^{\infty} g_{1}(\xi) f(x(t-\xi), v(t-\xi)) v(t-\xi) d \xi \\
& +f\left(x_{1}, v_{1}\right) v_{1} \int_{0}^{\infty} g_{1}(\xi) \ln \frac{f(x(t-\xi), v(t-\xi)) v(t-\xi)}{f(x(t), v(t)) v(t)} d \xi \\
& +\frac{\delta}{\alpha_{1}} y(t)-\frac{\delta}{\alpha_{1}} \int_{0}^{\infty} g_{2}(\xi) y(t-\xi) d \xi+\frac{\delta}{\alpha_{1}} y_{1} \int_{0}^{\infty} g_{2}(\xi) \ln \frac{y(t-\xi)}{y(t)} d \xi \\
= & d\left(1-\frac{f\left(x_{1}, v_{1}\right)}{f\left(x(t), v_{1}\right)}\right)\left(x_{1}-x(t)\right)+f\left(x_{1}, v_{1}\right) v_{1}-f\left(x_{1}, v_{1}\right) \frac{f\left(x_{1}, v_{1}\right) v_{1}}{f\left(x(t), v_{1}\right)} \\
& +\frac{f\left(x_{1}, v_{1}\right)}{f\left(x(t), v_{1}\right)} f(x(t), v(t)) v(t)-\frac{y_{1}}{y(t)} \int_{0}^{\infty} g_{1}(\xi) f(x(t-\xi), v(t-\xi)) v(t-\xi) d \xi
\end{aligned}
$$




$$
\begin{aligned}
& +\frac{\delta}{\alpha_{1}} y_{1}+\frac{p}{\alpha_{1}} y_{1} z(t)-\frac{\delta c}{\alpha_{1} \alpha_{2}} v(t)-\frac{\delta v_{1}}{\alpha_{1} v(t)} \int_{0}^{\infty} g_{2}(\xi) y(t-\xi) d \xi+\frac{\delta c v_{1}}{\alpha_{1} \alpha_{2}}+\frac{\delta q v_{1}}{\alpha_{1} \alpha_{2}} a(t) \\
& -\frac{p \gamma}{\alpha_{1} \beta} z(t)-\frac{\delta q b}{\alpha_{1} \alpha_{2} g} a(t)+f\left(x_{1}, v_{1}\right) v_{1} \int_{0}^{\infty} g_{1}(\xi) \ln \frac{f(x(t-\xi), v(t-\xi)) v(t-\xi)}{f(x(t), v(t)) v(t)} d \xi \\
& +\frac{\delta}{\alpha_{1}} y_{1} \int_{0}^{\infty} g_{2}(\xi) \ln \frac{y(t-\xi)}{y(t)} d \xi \\
= & d\left(1-\frac{f\left(x_{1}, v_{1}\right)}{f\left(x(t), v_{1}\right)}\right)\left(x_{1}-x(t)\right)+f\left(x_{1}, v_{1}\right) v_{1}\left[\int _ { 0 } ^ { \infty } g _ { 1 } ( \xi ) \left(-g\left(\frac{f\left(x_{1}, v_{1}\right)}{f\left(x(t), v_{1}\right)}\right)\right.\right. \\
& -g\left(\frac{f\left(x(t), v_{1}\right)}{f(x(t), v(t))}\right)-g\left(\frac{f(x(t-\xi), v(t-\xi)) v(t-\xi) y_{1}}{f\left(x_{1}, v_{1}\right) v_{1} y(t)}\right) d \xi \\
& \left.+\int_{0}^{\infty} g_{2}(\xi)\left(-g\left(\frac{v_{1} y(t-\xi)}{v(t)}\right)\right) d \xi\right]+\frac{p \gamma}{\alpha_{1} \beta}\left(\frac{\beta}{\gamma} y_{1}-1\right) z(t)+\frac{\delta q b}{\alpha_{1} \alpha_{2} g}\left(\frac{g}{b} v_{1}-1\right) a(t) \\
& +\frac{f\left(x_{1}, v_{1}\right)}{f(x(t), v(t)) f\left(x(t), v_{1}\right)}\left(f(x(t), v(t))-f\left(x(t), v_{1}\right)\right)\left(f(x(t), v(t)) v(t)-f\left(x(t), v_{1}\right) v_{1}\right) .
\end{aligned}
$$

From $\left(A_{2}\right)$, since $f(x, v)$ is strictly monotonically increasing with respect to $x$, we have

$$
d\left(1-\frac{f\left(x_{1}, v_{1}\right)}{f\left(x(t), v_{1}\right)}\right)\left(x_{1}-x(t)\right) \leqslant 0
$$

In addition, it follows from $\left(A_{3}\right)$ and $\left(A_{4}\right)$ that $f(x, v)$ is monotonically decreasing and $f(x, v) v$ is monotonically increasing with respect to $v$. This yields

$$
\left(f(x(t), v(t))-f\left(x(t), v_{1}\right)\right)\left(f(x(t), v(t)) v(t)-f\left(x(t), v_{1}\right) v_{1}\right) \leqslant 0 .
$$

Moreover, only if $v(t)=v_{1}$, we have $\left(f(x(t), v(t))-f\left(x(t), v_{1}\right)\right)\left(f(x(t), v(t)) v(t)-f\left(x(t), v_{1}\right) v_{1}\right)=0$. Furthermore, we have $\frac{\beta}{\gamma} y_{1}-1 \leqslant 0$ if $\mathfrak{R}_{1} \leqslant 1$, and $\frac{\mathrm{g}}{\mathrm{c}} v_{1}-1 \leqslant 0$ if $\mathfrak{R}_{2} \leqslant 1$.

Consequently, one can see that $V_{2}^{\prime}(t) \leqslant 0$, and $V_{2}^{\prime}(t)=0$ if $x(t)=x_{1}$ and $y(t)=y_{1}$. Hence, every solution of (2.2) tends to $M_{1}$, where $M_{1}$ is the largest invariant subset of $\left\{(x, y, v, z, a) \in \Gamma: V_{2}^{\prime}=0\right\}$. It can be easily verified that $M_{1}$ is singleton $\left\{E_{1}\right\}$ because every element of $M_{1}$ satisfies $v(t)=v_{1}$ and $z(t)=a(t)=0$ for all $t>0$. From the LaSalle invariance principle (see, e.g., Kuang [13, Corollary 5.2]), $E_{1}$ is globally attractive. In addition, since $V_{2}$ is estimated by a positive definite function from below:

$$
v_{2}(t) \geqslant u_{x_{1}}(t)+\frac{1}{\alpha_{1}} y_{1} g\left(\frac{y(t)}{y_{1}}\right)+\frac{\delta}{\alpha_{1} \alpha_{2}} v_{1} g\left(\frac{v(t)}{v_{1}}\right)+\frac{p}{\alpha_{1} \beta} z(t)+\frac{\delta q}{\alpha_{1} \alpha_{2} g} a(t),
$$

$E_{1}$ is uniformly stable. This implies that $E_{1}$ is globally asymptotically stable.

The following theorem indicates that the infection becomes chronic with persistent CTL immune response, but the viral load cannot activate the antibody immune responses.

Theorem 3.3. When $\mathfrak{R}_{1}>1$ and $\mathfrak{R}_{4} \leqslant 1$, the infection equilibrium $\mathrm{E}_{2}$ with only CTL immune response is globally asymptotically stable.

Proof. We will make use of the equations for the elements of $E_{2}$ in order to simplify the expressions:

$$
\left\{\begin{array}{l}
\lambda=d x_{2}+f\left(x_{2}, v_{2}\right) v_{2} \\
\alpha_{1} f\left(x_{2}, v_{2}\right) v_{2}=\delta y_{2}+p y_{2} z_{2}, \\
\alpha_{2} y_{2}=c v_{2} \\
\beta y_{2} z_{2}=\gamma z_{2} .
\end{array}\right.
$$

Define the following Lyapunov functional:

$$
\begin{aligned}
V_{3}(t)= & u_{x_{2}}(t)+\frac{1}{\alpha_{1}} y_{2} g\left(\frac{y(t)}{y_{2}}\right)+\left(\frac{\delta}{\alpha_{1} \alpha_{2}}+\frac{p z_{2}}{\alpha_{1} \alpha_{2}}\right) v_{2} g\left(\frac{v(t)}{v_{2}}\right)+\frac{p}{\alpha_{1} \beta} z_{2} g\left(\frac{z(t)}{z_{2}}\right)+\left(\frac{\delta q}{\alpha_{1} \alpha_{2} g}+\frac{p q z_{2}}{\alpha_{1} \alpha_{2} g}\right) a(t) \\
& +W_{3}(t)+W_{4}(t)
\end{aligned}
$$


where

$$
\begin{aligned}
& \mathrm{u}_{x_{2}}(\mathrm{t})=x(\mathrm{t})-\mathrm{x}_{2}-\int_{\mathrm{x}_{2}}^{x(t)} \frac{f\left(x_{2}, v_{2}\right)}{f\left(s, v_{2}\right)} d s, \\
& W_{3}(t)=f\left(x_{2}, v_{2}\right) v_{2} \int_{0}^{\infty} H_{1}(\xi) g\left(\frac{f(x(t-\xi), v(t-\xi)) v(t-\xi)}{f\left(x_{2}, v_{2}\right) v_{2}}\right) d \xi, \\
& W_{4}(t)=f\left(x_{2}, v_{2}\right) v_{2} \int_{0}^{\infty} H_{2}(\xi) g\left(\frac{y(t-\xi)}{y_{2}}\right) d \xi .
\end{aligned}
$$

We then obtain

$$
\begin{aligned}
W_{3}^{\prime}(t)= & f(x(t), v(t)) v(t)-\int_{0}^{\infty} g_{1}(\xi) f(x(t-\xi), v(t-\xi)) v(t-\xi) d \xi \\
& +f\left(x_{2}, v_{2}\right) v_{2} \int_{0}^{\infty} g_{1}(\xi) \ln \frac{f(x(t-\xi), v(t-\xi)) v(t-\xi)}{f(x(t), v(t)) v(t)} d \xi \\
W_{4}^{\prime}(t)= & \frac{\delta}{\alpha_{1}} y(t)-\frac{\delta}{\alpha_{1}} \int_{0}^{\infty} g_{2}(\xi) y(t-\xi) d \xi+\frac{p z_{2}}{\alpha_{1}} y(t) \\
& -\frac{p z_{2}}{\alpha_{1}} \int_{0}^{\infty} g_{2}(\xi) y(t-\xi) d \xi+f\left(x_{2}, v_{2}\right) v_{2} \int_{0}^{\infty} g_{2}(\xi) \ln \frac{y(t-\xi)}{y(t)} d \xi
\end{aligned}
$$

We calculate the time derivative of $V_{3}(t)$ :

$$
\begin{aligned}
V_{3}^{\prime}(t)= & \left(1-\frac{f\left(x_{2}, v_{2}\right)}{f\left(x(t), v_{2}\right)}\right)(\lambda-d x(t)-f(x(t), v(t)) v(t)) \\
& +\frac{1}{\alpha_{1}}\left(1-\frac{y_{2}}{y(t)}\right)\left(\alpha_{1} \int_{0}^{\infty} g_{1}(\xi) f(x(t-\xi), v(t-\xi)) v(t-\xi) d \xi-\delta y(t)-p y(t) z(t)\right) \\
& +\left(\frac{\delta}{\alpha_{1} \alpha_{2}}+\frac{p z_{2}}{\alpha_{1} \alpha_{2}}\right)\left(1-\frac{v_{2}}{v(t)}\right)\left(\alpha_{2} \int_{0}^{\infty} g_{2}(\xi) y(t-\xi) d \xi-c v(t)-q a(t) v(t)\right) \\
& +\frac{p}{\alpha_{1} \beta}\left(1-\frac{z_{2}}{z(t)}\right)(\beta y(t) z(t)-\gamma z(t))+\left(\frac{\delta q}{\alpha_{1} \alpha_{2} g}+\frac{p q z_{2}}{\alpha_{1} \alpha_{2} g}\right)(g a(t) v(t)-b a(t)) \\
& +f(x(t), v(t)) v(t)-\int_{0}^{\infty} g_{1}(\xi) f(x(t-\xi), v(t-\xi)) v(t-\xi) d \xi \\
& +f\left(x_{2}, v_{2}\right) v_{2} \int_{0}^{\infty} g_{1}(\xi) \ln \frac{f(x(t-\xi), v(t-\xi)) v(t-\xi)}{f(x(t), v(t)) v(t)} d \xi \\
& +\frac{\delta}{\alpha_{1}} y(t)-\frac{\delta}{\alpha_{1}} \int_{0}^{\infty} g_{2}(\xi) y(t-\xi) d \xi+\frac{p z_{2}}{\alpha_{1}} y(t) \\
& -\frac{p z_{2}}{\alpha_{1}} \int_{0}^{\infty} g_{2}(\xi) y(t-\xi) d \xi+f\left(x_{2}, v_{2}\right) v_{2} \int_{0}^{\infty} g_{2}(\xi) \ln \frac{y(t-\xi)}{y(t)} d \xi \\
= & d\left(1-\frac{f\left(x_{2}, v_{2}\right)}{f\left(x(t), v_{2}\right)}\right)\left(x_{2}-x(t)\right)+f\left(x_{2}, v_{2}\right) v_{2}-f\left(x_{2}, v_{2}\right) \frac{f\left(x_{2}, v_{2}\right) v_{2}}{f\left(x(t), v_{2}\right)} \\
& +\frac{f\left(x_{2}, v_{2}\right)}{f\left(x(t), v_{2}\right)} f(x(t), v(t)) v(t)-\frac{y 2}{y(t)} \int_{0}^{\infty} g_{1}(\xi) f(x(t-\xi), v(t-\xi)) v(t-\xi) d \xi \\
& +\frac{\delta}{\alpha_{1}} y_{2}-\frac{\delta c}{\alpha_{1} \alpha_{2}} v(t)-\frac{\delta v_{2}}{\alpha_{1} v(t)} \int_{0}^{\infty} g_{2}(\xi) y(t-\xi) d \xi+\frac{\delta c v_{2}}{\alpha_{1} \alpha_{2}}+\frac{\delta q v_{2}}{\alpha_{1} \alpha_{2}} a(t) \\
& -\frac{p z_{2} c}{\alpha_{1} \alpha_{2}} v(t)-\frac{p z_{2} v_{2}}{\alpha_{1} v(t)} \int_{0}^{\infty} g_{2}(\xi) y(t-\xi) d \xi+\frac{p c z_{2} v_{2}}{\alpha_{1} \alpha_{2}}+\frac{p q z_{2} v_{2}}{\alpha_{1} \alpha_{2}} a(t) \\
& +\frac{p \gamma z_{2}}{\alpha_{1} \beta}-\frac{b \delta q}{\alpha_{1} \alpha_{2} g} a(t)-\frac{b p q z_{2}}{\alpha_{1} \alpha_{2} g} a(t) \\
& +f\left(x_{2}, v_{2}\right) v_{2} \int_{0}^{\infty} g_{1}(\xi) \ln \frac{f(x(t-\xi), v(t-\xi)) v(t-\xi)}{f(x), v(t)) v(t)} d \xi \\
&
\end{aligned}
$$




$$
\begin{aligned}
& +f\left(x_{2}, v_{2}\right) v_{2} \int_{0}^{\infty} g_{2}(\xi) \ln \frac{y(t-\xi)}{y(t)} d \xi \\
= & d\left(1-\frac{f\left(x_{2}, v_{2}\right)}{f\left(x(t), v_{2}\right)}\right)\left(x_{2}-x(t)\right)+f\left(x_{2}, v_{2}\right) v_{2}\left[\int _ { 0 } ^ { \infty } g _ { 1 } ( \xi ) \left(-g\left(\frac{f\left(x_{2}, v_{2}\right)}{f\left(x(t), v_{2}\right)}\right)\right.\right. \\
& \left.-g\left(\frac{f\left(x(t), v_{2}\right)}{f(x(t), v(t))}\right)-g\left(\frac{f(x(t-\xi), v(t-\xi)) v(t-\xi) y_{2}}{f\left(x_{2}, v_{2}\right) v_{2} y(t)}\right)\right) d \xi \\
& \left.+\int_{0}^{\infty} g_{2}(\xi)\left(-g\left(\frac{v_{2} y(t-\xi)}{v(t) y_{2}}\right)\right) d \xi\right]+\frac{b q}{\alpha_{1} \alpha_{2} g}\left(\delta+p z_{2}\right)\left(\frac{\alpha_{2} g \gamma}{\beta b c}-1\right) a(t) \\
& +\frac{f\left(x_{2}, v_{2}\right)}{f(x(t), v(t)) f\left(x(t), v_{2}\right)}\left(f(x(t), v(t))-f\left(x(t), v_{2}\right)\right)\left(f(x(t), v(t)) v(t)-f\left(x(t), v_{2}\right) v_{2}\right) .
\end{aligned}
$$

From $\left(A_{2}\right)$, since $f(x, v)$ is strictly monotonically increasing with respect to $x$, we have

$$
d\left(1-\frac{f\left(x_{2}, v_{2}\right)}{f\left(x(t), v_{2}\right)}\right)\left(x_{2}-x(t)\right) \leqslant 0
$$

It follows from $\left(A_{3}\right)$ and $\left(A_{4}\right)$ that $f(x, v)$ is monotonically decreasing and $f(x, v) v$ is monotonically increasing with respect to $v$, we obtain

$$
\left(f(x(t), v(t))-f\left(x(t), v_{2}\right)\right)\left(f(x(t), v(t)) v(t)-f\left(x(t), v_{2}\right) v_{2}\right) \leqslant 0 .
$$

Moreover, only if $v(t)=v_{2}$, we have $\left(f(x(t), v(t))-f\left(x(t), v_{2}\right)\right)\left(f(x(t), v(t)) v(t)-f\left(x(t), v_{2}\right) v_{2}\right)=0$. We here note that $\frac{\alpha_{2} g \gamma}{\beta b c}-1 \leqslant 0$ if $\Re_{4} \leqslant 1$.

Consequently, one can see that $V_{3}^{\prime}(t) \leqslant 0$, and $V_{3}^{\prime}(t)=0$ if $x(t)=x_{2}$ and $y(t)=y_{2}$. Hence, every solution of (2.2) tends to $M_{2}$, where $M_{2}$ is the largest invariant subset of $\left\{(x, y, v, z, a) \in \Gamma: V_{3}^{\prime}=0\right\}$. It can be easily verified that $M_{2}$ is singleton $\left\{E_{2}\right\}$ because every element of $M_{2}$ satisfies $v(t)=v_{2}, z(t)=z_{2}$ and $a(t)=0$ for all $t>0$. From the LaSalle invariance principle (see, e.g., Kuang [13, Corollary 5.2]), $E_{2}$ is globally attractive. In addition, since $V_{3}$ is estimated by a positive definite function from below:

$$
v_{3}(t) \geqslant u_{x_{2}}(t)+\frac{1}{\alpha_{1}} y_{2} g\left(\frac{y(t)}{y_{2}}\right)+\left(\frac{\delta}{\alpha_{1} \alpha_{2}}+\frac{p z_{2}}{\alpha_{1} \alpha_{2}}\right) v_{2} g\left(\frac{v(t)}{v_{2}}\right)+\frac{p}{\alpha_{1} \beta} z_{2} g\left(\frac{z(t)}{z_{2}}\right)+\left(\frac{\delta q}{\alpha_{1} \alpha_{2} g}+\frac{p q z_{2}}{\alpha_{1} \alpha_{2} g}\right) a(t),
$$

$E_{2}$ is uniformly stable. This implies that $E_{2}$ is globally asymptotically stable.

The following theorem indicates that the infection becomes chronic with persistent antibody immune response, but the infected cells can not stimulate and activate CTL immune responses.

Theorem 3.4. When $\mathfrak{R}_{2}>1$ and $\mathfrak{R}_{3} \leqslant 1$, the infection equilibrium $\mathrm{E}_{3}$ with only antibody immune response is globally asymptotically stable.

Proof. We will make use of the equations for the elements of $E_{3}$ in order to simplify the expressions:

$$
\left\{\begin{array}{l}
\lambda=d x_{3}+f\left(x_{3}, v_{3}\right) v_{3} \\
\alpha_{1} f\left(x_{3}, v_{3}\right) v_{3}=\delta y_{3} \\
\alpha_{2} y_{3}=c v_{3}+q a_{3} v_{3} \\
g a_{3} v_{3}=b a_{3}
\end{array}\right.
$$

Define the following Lyapunov functional:

$$
\mathrm{V}_{4}(\mathrm{t})=\mathrm{u}_{\mathrm{x}_{3}}(\mathrm{t})+\frac{1}{\alpha_{1}} \mathrm{y}_{3} g\left(\frac{\mathrm{y}(\mathrm{t})}{\mathrm{y}_{3}}\right)+\frac{\delta}{\alpha_{1} \alpha_{2}} v_{3} g\left(\frac{v(t)}{v_{3}}\right)+\frac{p}{\alpha_{1} \beta} z(t)+\frac{\delta q}{\alpha_{1} \alpha_{2} g} a_{3} g\left(\frac{a(t)}{a_{3}}\right)+W_{5}(t)+W_{6}(t),
$$

where

$$
u_{x_{3}}(t)=x(t)-x_{3}-\int_{x_{3}}^{x(t)} \frac{f\left(x_{3}, v_{3}\right)}{f\left(s, v_{3}\right)} d s,
$$




$$
\begin{aligned}
& W_{5}(t)=f\left(x_{3}, v_{3}\right) v_{3} \int_{0}^{\infty} H_{1}(\xi) g\left(\frac{f(x(t-\xi), v(t-\xi)) v(t-\xi)}{f\left(x_{3}, v_{3}\right) v_{3}}\right) d \xi \\
& W_{6}(t)=f\left(x_{3}, v_{3}\right) v_{3} \int_{0}^{\infty} H_{2}(\xi) g\left(\frac{y(t-\xi)}{y_{3}}\right) d \xi
\end{aligned}
$$

We then obtain

$$
\begin{aligned}
W_{5}^{\prime}(t)= & f(x(t), v(t)) v(t)-\int_{0}^{\infty} g_{1}(\xi) f(x(t-\xi), v(t-\xi)) v(t-\xi) d \xi \\
& +f\left(x_{3}, v_{3}\right) v_{3} \int_{0}^{\infty} g_{1}(\xi) \ln \frac{f(x(t-\xi), v(t-\xi)) v(t-\xi)}{f(x(t), v(t)) v(t)} d \xi \\
W_{6}^{\prime}(t)= & \frac{\delta}{\alpha_{1}} y(t)-\frac{\delta}{\alpha_{1}} \int_{0}^{\infty} g_{2}(\xi) y(t-\xi) d \xi+f\left(x_{3}, v_{3}\right) v_{3} \int_{0}^{\infty} g_{2}(\xi) \ln \frac{y(t-\xi)}{y(t)} d \xi .
\end{aligned}
$$

We calculate the time derivative of $V_{4}(t)$ :

$$
\begin{aligned}
& V_{4}^{\prime}(t)=\left(1-\frac{f\left(x_{3}, v_{3}\right)}{f\left(x(t), v_{3}\right)}\right)(\lambda-d x(t)-f(x(t), v(t)) v(t)) \\
& +\frac{1}{\alpha_{1}}\left(1-\frac{y_{3}}{y(t)}\right)\left(\alpha_{1} \int_{0}^{\infty} g_{1}(\xi) f(x(t-\xi), v(t-\xi)) v(t-\xi) d \xi-\delta y(t)-p y(t) z(t)\right) \\
& +\frac{\delta}{\alpha_{1} \alpha_{2}}\left(1-\frac{v_{3}}{v(t)}\right)\left(\alpha_{2} \int_{0}^{\infty} g_{2}(\xi) y(t-\xi) d \xi-c v(t)-q a(t) v(t)\right) \\
& +\frac{p}{\alpha_{1} \beta}(\beta y(t) z(t)-\gamma z(t))+\frac{\delta q}{\alpha_{1} \alpha_{2} g}\left(1-\frac{a_{3}}{a(t)}\right)(g a(t) v(t)-b a(t)) \\
& +f(x(t), v(t)) v(t)-\int_{0}^{\infty} g_{1}(\xi) f(x(t-\xi), v(t-\xi)) v(t-\xi) d \xi \\
& +f\left(x_{3}, v_{3}\right) v_{3} \int_{0}^{\infty} g_{1}(\xi) \ln \frac{f(x(t-\xi), v(t-\xi)) v(t-\xi)}{f(x(t), v(t)) v(t)} d \xi \\
& +\frac{\delta}{\alpha_{1}} y(t)-\frac{\delta}{\alpha_{1}} \int_{0}^{\infty} g_{2}(\xi) y(t-\xi) d \xi+f\left(x_{3}, v_{3}\right) v_{3} \int_{0}^{\infty} g_{2}(\xi) \ln \frac{y(t-\xi)}{y(t)} d \xi \\
& =d\left(1-\frac{f\left(x_{3}, v_{3}\right)}{f\left(x(t), v_{3}\right)}\right)\left(x_{3}-x(t)\right)+f\left(x_{3}, v_{3}\right) v_{3}-f\left(x_{3}, v_{3}\right) \frac{f\left(x_{3}, v_{3}\right) v_{3}}{f\left(x(t), v_{3}\right)} \\
& +\frac{f\left(x_{3}, v_{3}\right)}{f\left(x(t), v_{3}\right)} f(x(t), v(t)) v(t)-\frac{y_{3}}{y(t)} \int_{0}^{\infty} g_{1}(\xi) f(x(t-\xi), v(t-\xi)) v(t-\xi) d \xi \\
& +\frac{\delta y_{3}}{\alpha_{1}}+\frac{p y_{3}}{\alpha_{1}} z(t)-\frac{\delta c}{\alpha_{1} \alpha_{2}} v(t)-\frac{\delta v_{3}}{\alpha_{1} v(t)} \int_{0}^{\infty} g_{2}(\xi) y(t-\xi) d \xi+\frac{\delta c v_{3}}{\alpha_{1} \alpha_{2}}-\frac{p \gamma}{\alpha_{1} \beta} z(t) \\
& -\frac{\delta q a_{3}}{\alpha_{1} \alpha_{2}} v(t)+\frac{b \delta q a_{3}}{\alpha_{1} \alpha_{2} g}+f\left(x_{3}, v_{3}\right) v_{3} \int_{0}^{\infty} g_{1}(\xi) \ln \frac{f(x(t-\xi), v(t-\xi)) v(t-\xi)}{f(x(t), v(t)) v(t)} d \xi \\
& +f\left(x_{3}, v_{3}\right) v_{3} \int_{0}^{\infty} g_{2}(\xi) \ln \frac{y(t-\xi)}{y(t)} d \xi \\
& =d\left(1-\frac{f\left(x_{3}, v_{3}\right)}{f\left(x(t), v_{3}\right)}\right)\left(x_{3}-x(t)\right)+f\left(x_{3}, v_{3}\right) v_{3}\left[\int _ { 0 } ^ { \infty } g _ { 1 } ( \xi ) \left(-g\left(\frac{f\left(x_{3}, v_{3}\right)}{f\left(x(t), v_{3}\right)}\right)\right.\right. \\
& \left.-g\left(\frac{f\left(x(t), v_{3}\right)}{f(x(t), v(t))}\right)-g\left(\frac{f(x(t-\xi), v(t-\xi)) v(t-\xi) y_{3}}{f\left(x_{3}, v_{3}\right) v_{3} y(t)}\right)\right) d \xi \\
& \left.+\int_{0}^{\infty} g_{2}(\xi)\left(-g\left(\frac{\nu_{3} y(t-\xi)}{v(t) y_{3}}\right)\right) d \xi\right]+\frac{p \gamma}{\alpha_{1} \beta}\left(\frac{\beta y_{3}}{\gamma}-1\right) z(t) \\
& +\frac{f\left(x_{3}, v_{3}\right)}{f(x(t), v(t)) f\left(x(t), v_{3}\right)}\left(f(x(t), v(t))-f\left(x(t), v_{3}\right)\right)\left(f(x(t), v(t)) v(t)-f\left(x(t), v_{3}\right) v_{3}\right) .
\end{aligned}
$$


From $\left(A_{2}\right)$, since $f(x, v)$ is strictly monotonically increasing with respect to $x$, we have

$$
d\left(1-\frac{f\left(x_{3}, v_{3}\right)}{f\left(x(t), v_{3}\right)}\right)\left(x_{3}-x(t)\right) \leqslant 0
$$

It follows from $\left(A_{3}\right)$ and $\left(A_{4}\right)$ that $f(x, v)$ is monotonically decreasing and $f(x, v) v$ is monotonically increasing with respect to $v$. This yields

$$
\left(f(x(t), v(t))-f\left(x(t), v_{3}\right)\right)\left(f(x(t), v(t)) v(t)-f\left(x(t), v_{3}\right) v_{3}\right) \leqslant 0 .
$$

Moreover, only if $v(t)=v_{3}$, we have $\left(f(x(t), v(t))-f\left(x(t), v_{3}\right)\right)\left(f(x(t), v(t)) v(t)-f\left(x(t), v_{3}\right) v_{3}\right)=0$. Furthermore, $\frac{\beta}{\gamma} y_{3}-1 \leqslant 0$ if $\Re_{3} \leqslant 1$.

Consequently, one can see that $V_{4}^{\prime}(t) \leqslant 0$, and $V_{4}^{\prime}(t)=0$ if $x(t)=x_{3}$ and $y(t)=y_{3}$. Hence, every solution of (2.2) tends to $M_{3}$, where $M_{3}$ is the largest invariant subset of $\left\{(x, y, v, z, a) \in \Gamma: V_{4}^{\prime}=0\right\}$. It can be easily verified that $M_{3}$ is singleton $\left\{E_{3}\right\}$ because every element of $M_{3}$ satisfies $v(t)=v_{3}, z(t)=0$ and $a(t)=a_{3}$ for all $t>0$. From the LaSalle invariance principle (see, e.g., Kuang [13, Corollary 5.2]), $E_{3}$ is globally attractive. In addition, since $V_{4}$ is estimated by a positive definite function from below:

$$
v_{4}(t) \geqslant u_{x_{3}}(t)+\frac{1}{\alpha_{1}} y_{3} g\left(\frac{y(t)}{y_{3}}\right)+\frac{\delta}{\alpha_{1} \alpha_{2}} v_{3} g\left(\frac{v(t)}{v_{3}}\right)+\frac{p}{\alpha_{1} \beta} z(t)+\frac{\delta q}{\alpha_{1} \alpha_{2} g} a_{3} g\left(\frac{a(t)}{a_{3}}\right),
$$

$E_{3}$ is uniformly stable. This implies that $E_{3}$ is globally asymptotically stable.

The following theorem indicates that susceptible cells, infected cells, free virus particles, CTLs and antibodies eventually coexist in vivo.

Theorem 3.5. When $\mathfrak{R}_{3}>1$ and $\mathfrak{R}_{4}>1$, the infection equilibrium $\mathrm{E}_{4}$ with both CTL response and antibody response is globally asymptotically stable.

Proof. We will make use of the equations for the elements of $E_{4}$ in order to simplify the expressions:

$$
\left\{\begin{array}{l}
\lambda=d x_{4}+f\left(x_{4}, v_{4}\right) v_{4}, \\
\alpha_{1} f\left(x_{4}, v_{4}\right) v_{4}=\delta y_{4}+p y_{4} z_{4}, \\
\alpha_{2} y_{4}=c v_{4}+q a_{4} v_{4}, \\
\beta y_{4} z_{4}=\gamma z_{4}, \\
g a_{4} v_{4}=b a_{4} .
\end{array}\right.
$$

Define the following Lyapunov functional:

$$
\begin{aligned}
\mathrm{v}_{5}(\mathrm{t})= & \mathrm{U}_{x_{4}}(\mathrm{t})+\frac{1}{\alpha_{1}} \mathrm{y}_{4} \mathrm{~g}\left(\frac{\mathrm{y}(\mathrm{t})}{\mathrm{y}_{4}}\right)+\left(\frac{\delta}{\alpha_{1} \alpha_{2}}+\frac{\mathrm{p} z_{4}}{\alpha_{1} \alpha_{2}}\right) v_{4} g\left(\frac{v(t)}{v_{4}}\right) \\
& +\frac{p}{\alpha_{1} \beta} z_{4} g\left(\frac{z(t)}{z_{4}}\right)+\left(\frac{\delta \mathrm{q}}{\alpha_{1} \alpha_{2} g}+\frac{p q z_{4}}{\alpha_{1} \alpha_{2} g}\right) a_{4} g\left(\frac{\mathrm{a}(\mathrm{t})}{a_{4}}\right)+W_{7}(t)+W_{8}(t),
\end{aligned}
$$

where

$$
\begin{aligned}
& \mathrm{u}_{x_{4}}(\mathrm{t})=x(\mathrm{t})-x_{4}-\int_{x_{4}}^{x(t)} \frac{f\left(x_{4}, v_{4}\right)}{f\left(s, v_{4}\right)} d s \\
& W_{7}(t)=f\left(x_{4}, v_{4}\right) v_{4} \int_{0}^{\infty} H_{1}(\xi) g\left(\frac{f(x(t-\xi), v(t-\xi)) v(t-\xi)}{f\left(x_{4}, v_{4}\right) v_{4}}\right) d \xi \\
& W_{8}(t)=f\left(x_{4}, v_{4}\right) v_{4} \int_{0}^{\infty} H_{2}(\xi) g\left(\frac{y(t-\xi)}{y_{4}}\right) d \xi
\end{aligned}
$$


We then obtain

$$
\begin{aligned}
W_{7}^{\prime}(t)= & f(x(t), v(t)) v(t)-\int_{0}^{\infty} g_{1}(\xi) f(x(t-\xi), v(t-\xi)) v(t-\xi) d \xi \\
& +f\left(x_{4}, v_{4}\right) v_{4} \int_{0}^{\infty} g_{1}(\xi) \ln \frac{f(x(t-\xi), v(t-\xi)) v(t-\xi)}{f(x(t), v(t)) v(t)} d \xi, \\
W_{8}^{\prime}(t)= & \frac{\delta}{\alpha_{1}} y(t)-\frac{\delta}{\alpha_{1}} \int_{0}^{\infty} g_{2}(\xi) y(t-\xi) d \xi+\frac{p z_{4}}{\alpha_{1}} y(t) \\
& -\frac{p z_{4}}{\alpha_{1}} \int_{0}^{\infty} g_{2}(\xi) y(t-\xi) d \xi+f\left(x_{4}, v_{4}\right) v_{4} \int_{0}^{\infty} g_{2}(\xi) \ln \frac{y(t-\xi)}{y(t)} d \xi .
\end{aligned}
$$

We calculate the time derivative of $V_{5}(t)$ :

$$
\begin{aligned}
& V_{5}^{\prime}(t)=\left(1-\frac{f\left(x_{4}, v_{4}\right)}{f\left(x(t), v_{4}\right)}\right)(\lambda-d x(t)-f(x(t), v(t)) v(t)) \\
& +\frac{1}{\alpha_{1}}\left(1-\frac{y_{4}}{y(t)}\right)\left(\alpha_{1} \int_{0}^{\infty} g_{1}(\xi) f(x(t-\xi), v(t-\xi)) v(t-\xi) d \xi-\delta y(t)-p y(t) z(t)\right) \\
& +\left(\frac{\delta}{\alpha_{1} \alpha_{2}}+\frac{p z_{4}}{\alpha_{1} \alpha_{2}}\right)\left(1-\frac{v_{4}}{v(t)}\right)\left(\alpha_{2} \int_{0}^{\infty} g_{2}(\xi) y(t-\xi) d \xi-c v(t)-q a(t) v(t)\right) \\
& +\frac{p}{\alpha_{1} \beta}\left(1-\frac{z_{4}}{z(t)}\right)(\beta y(t) z(t)-\gamma z(t)) \\
& +\left(\frac{\delta q}{\alpha_{1} \alpha_{2} g}+\frac{p q z_{4}}{\alpha_{1} \alpha_{2} g}\right)\left(1-\frac{a_{4}}{a(t)}\right)(g a(t) v(t)-b a(t)) \\
& +f(x(t), v(t)) v(t)-\int_{0}^{\infty} g_{1}(\xi) f(x(t-\xi), v(t-\xi)) v(t-\xi) d \xi \\
& +f\left(x_{4}, v_{4}\right) v_{4} \int_{0}^{\infty} g_{1}(\xi) \ln \frac{f(x(t-\xi), v(t-\xi)) v(t-\xi)}{f(x(t), v(t)) v(t)} d \xi \\
& +\frac{\delta}{\alpha_{1}} y(t)-\frac{\delta}{\alpha_{1}} \int_{0}^{\infty} g_{2}(\xi) y(t-\xi) d \xi+\frac{p z_{4}}{\alpha_{1}} y(t) \\
& -\frac{p z_{4}}{\alpha_{1}} \int_{0}^{\infty} g_{2}(\xi) y(t-\xi) d \xi+f\left(x_{4}, v_{4}\right) v_{4} \int_{0}^{\infty} g_{2}(\xi) \ln \frac{y(t-\xi)}{y(t)} d \xi \\
& =d\left(1-\frac{f\left(x_{4}, v_{4}\right)}{f\left(x(t), v_{4}\right)}\right)\left(x_{4}-x(t)\right)+f\left(x_{4}, v_{4}\right) v_{4}-f\left(x_{4}, v_{4}\right) \frac{f\left(x_{4}, v_{4}\right) v_{4}}{f\left(x(t), v_{4}\right)} \\
& +\frac{f\left(x_{4}, v_{4}\right)}{f\left(x(t), v_{4}\right)} f(x(t), v(t)) v(t)-\frac{y_{4}}{y(t)} \int_{0}^{\infty} g_{1}(\xi) f(x(t-\xi), v(t-\xi)) v(t-\xi) d \xi \\
& +\frac{\delta y_{4}}{\alpha_{1}}-\frac{\delta c}{\alpha_{1} \alpha_{2}} v(t)-\frac{\delta v_{4}}{\alpha_{1} v(t)} \int_{0}^{\infty} g_{2}(\xi) y(t-\xi) d \xi+\frac{\delta c v_{4}}{\alpha_{1} \alpha_{2}} \\
& -\frac{p z_{4} c}{\alpha_{1} \alpha_{2}} v(t)-\frac{p z_{4} v_{4}}{\alpha_{1} v(t)} \int_{0}^{\infty} g_{2}(\xi) y(t-\xi) d \xi+\frac{p c z_{4} v_{4}}{\alpha_{1} \alpha_{2}} \\
& +\frac{p \gamma z_{4}}{\alpha_{1} \beta}-\frac{q \delta a_{4}}{\alpha_{1} \alpha_{2}} v(t)+\frac{b q \delta a_{4}}{\alpha_{1} \alpha_{2} g}-\frac{p q z_{4} a_{4}}{\alpha_{1} \alpha_{2}} v(t)+\frac{b p q z_{4} a_{4}}{\alpha_{1} \alpha_{2} g} \\
& +f\left(x_{4}, v_{4}\right) v_{4} \int_{0}^{\infty} g_{1}(\xi) \ln \frac{f(x(t-\xi), v(t-\xi)) v(t-\xi)}{f(x(t), v(t)) v(t)} d \xi+f\left(x_{4}, v_{4}\right) v_{4} \int_{0}^{\infty} g_{2}(\xi) \ln \frac{y(t-\xi)}{y(t)} d \xi \\
& =d\left(1-\frac{f\left(x_{4}, v_{4}\right)}{f\left(x(t), v_{4}\right)}\right)\left(x_{4}-x(t)\right)+f\left(x_{4}, v_{4}\right) v_{4}\left[\int _ { 0 } ^ { \infty } g _ { 1 } ( \xi ) \left(-g\left(\frac{f\left(x_{4}, v_{4}\right)}{f\left(x(t), v_{4}\right)}\right)-g\left(\frac{f\left(x(t), v_{4}\right)}{f(x(t), v(t))}\right)\right.\right. \\
& \left.\left.-g\left(\frac{f(x(t-\xi), v(t-\xi)) v(t-\xi) y_{4}}{f\left(x_{4}, v_{4}\right) v_{4} y(t)}\right)\right) d \xi+\int_{0}^{\infty} g_{2}(\xi)\left(-g\left(\frac{v_{4} y(t-\xi)}{v(t) y_{4}}\right)\right) d \xi\right] \\
& +\frac{f\left(x_{4}, v_{4}\right)}{f(x(t), v(t)) f\left(x(t), v_{4}\right)}\left(f(x(t), v(t))-f\left(x(t), v_{4}\right)\right)\left(f(x(t), v(t)) v(t)-f\left(x(t), v_{4}\right) v_{4}\right) .
\end{aligned}
$$


From $\left(A_{2}\right)$, since $f(x, v)$ is strictly monotonically increasing with respect to $x$, we have

$$
d\left(1-\frac{f\left(x_{4}, v_{4}\right)}{f\left(x(t), v_{4}\right)}\right)\left(x_{4}-x(t)\right) \leqslant 0
$$

It also follows from $\left(A_{3}\right)$ and $\left(A_{4}\right)$ that $f(x, v)$ is monotonically decreasing and $f(x, v) v$ is monotonically increasing with respect to $v$. This yields

$$
\left(f(x(t), v(t))-f\left(x(t), v_{4}\right)\right)\left(f(x(t), v(t)) v(t)-f\left(x(t), v_{4}\right) v_{4}\right) \leqslant 0 .
$$

Moreover, only if $v(t)=v_{4}$, we have $\left(f(x(t), v(t))-f\left(x(t), v_{4}\right)\right)\left(f(x(t), v(t)) v(t)-f\left(x(t), v_{4}\right) v_{4}\right)=0$.

Consequently, one can see that $V_{5}^{\prime}(t) \leqslant 0$, and $V_{5}^{\prime}(t)=0$ if $x(t)=x_{4}$ and $y(t)=y_{4}$. Hence, every solution of (2.2) tends to $M_{4}$, where $M_{4}$ is the largest invariant subset of $\left\{(x, y, v, z, a) \in \Gamma: V_{5}^{\prime}=0\right\}$. It can be easily verified that $M_{4}$ is singleton $\left\{E_{4}\right\}$ because every element of $M_{4}$ satisfies $v(t)=v_{4}, z(t)=z_{4}$ and $a(t)=a_{4}$ for all $t>0$. From the LaSalle invariance principle (see, e.g., Kuang [13, Corollary 5.2]), $E_{4}$ is globally attractive. In addition, since $V_{5}$ is estimated by a positive definite function from below:

$$
\begin{aligned}
v_{5}(t) \geqslant & u_{x_{4}}(t)+\frac{1}{\alpha_{1}} y_{4} g\left(\frac{y(t)}{y_{4}}\right)+\left(\frac{\delta}{\alpha_{1} \alpha_{2}}+\frac{p z_{4}}{\alpha_{1} \alpha_{2}}\right) v_{4} g\left(\frac{v(t)}{v_{4}}\right) \\
& +\frac{p}{\alpha_{1} \beta} z_{4} g\left(\frac{z(t)}{z_{4}}\right)+\left(\frac{\delta q}{\alpha_{1} \alpha_{2} g}+\frac{p q z_{4}}{\alpha_{1} \alpha_{2} g}\right) a_{4} g\left(\frac{a(t)}{a_{4}}\right),
\end{aligned}
$$

$E_{4}$ is uniformly stable. This implies that $E_{4}$ is globally asymptotically stable.

\section{Concluding remarks}

Throughout this paper, the asymptotic behavior of the solutions of a virus model incorporating the effect of cell-mediated and humoral immune responses has been studied. By specifying the basic reproduction numbers of the model (2.2), we establish that an infection-free equilibrium $E_{0}$ is globally asymptotically stable if and only if $\mathfrak{R}_{0} \leqslant 1$ and that there are sufficient conditions under which each of four infection equilibria is globally asymptotically stable for $\mathfrak{R}_{0}>1$. Similar to the construction methods of Lyapunov functionals presented in Section 3, stability results similar to those in Theorems 3.2-3.5 are also established for the following model with "discrete" delays:

$$
\left\{\begin{array}{l}
x^{\prime}(t)=\lambda-d x(t)-f(x(t), v(t)) v(t) \\
y^{\prime}(t)=\alpha_{1} f\left(x\left(t-\tau_{1}\right), v\left(t-\tau_{1}\right)\right) v\left(t-\tau_{1}\right)-\delta y(t)-p y(t) z(t) \\
v^{\prime}(t)=\alpha_{2} y\left(t-\tau_{2}\right)-c v(t)-q a(t) v(t) \\
z^{\prime}(t)=\beta y(t) z(t)-\gamma z(t) \\
a^{\prime}(t)=g a(t) v(t)-b a(t)
\end{array}\right.
$$

From the relation $\mathfrak{R}_{4}=\frac{\mathfrak{R}_{2}}{\mathfrak{R}_{1}}$, we immediately derive the following corollary for system (4.1).

Corollary 4.1. The following statement holds true.

(i) When $\mathfrak{R}_{0} \leqslant 1$, the infection-free equilibrium $\mathrm{E}_{0}$ is globally asymptotically stable.

Under the condition $\mathfrak{R}_{0}>1$, the following statements also hold true.

(ii) When $\mathfrak{R}_{1} \leqslant 1$ and $\mathfrak{R}_{2} \leqslant 1$, the immune-free infection equilibrium $\mathrm{E}_{1}$ is globally asymptotically stable.

(iii) When $\mathfrak{R}_{1}>1$ and $\mathfrak{R}_{2} \leqslant \mathfrak{R}_{1}$, the infection equilibrium $\mathrm{E}_{2}$ with only CTL immune response is globally asymptotically stable.

(iv) When $\mathfrak{R}_{3} \leqslant 1$ and $\mathfrak{R}_{2}>1$, the infection equilibrium $\mathrm{E}_{3}$ with only humoral immune response is globally asymptotically stable. 
(v) When $\mathfrak{R}_{3}>1$ and $\mathfrak{R}_{2}>\mathfrak{R}_{1}$, the infection equilibrium $\mathrm{E}_{4}$ with both CTL response and humoral response is globally asymptotically stable.

To prove the global stability of the four infection equilibria $E_{i}(i=1, \cdots, 4)$, we construct Lyapunov functionals with the help of Volterra-type function $\mathrm{g}$. Moreover, the functional methods in Wang et al. [24, Section 3] are also extended to obtain the global dynamics when the incidence rate is non-separable with respect to uninfected cells and free virus particles. The analytical approach is motivated by the construction methods in $[9,11,15,24,29]$. The hypotheses $\left(\mathrm{A}_{1}\right)-\left(\mathrm{A}_{4}\right)$, including not only a bilinear incidence rate $f(x, v) v=k x v$, separable incidence rate $f(x, v) v=F(x) G(v)$ but also (non-separable) standard incidence rate $f(x, v) v=\frac{x v}{x+v}$ and Beddington-DeAngelis functional response $f(x, v) v=\frac{x v}{1+\alpha_{1} x+\alpha_{2} v}$, play a crucial role in determining the suitable Lyapunov functionals. Moreover, the hypothesis $\left(\mathrm{A}_{4}\right)$, indicating that the incidence rate $f(x, v) v$ is monotonically increasing with respect to free virus particles, is not necessary for the global stability of the infection-free equilibrium $E_{0}$. Finally, we note that the global stability for the four infection equilibria $E_{i}(i=1, \cdots, 4)$ is still completely determined. Biologically, the global stability of infection equilibrium $E_{2}, E_{3}, E_{4}$ indicates that lifelong immunity (CTL responses and antibody responses) can be achieved in the host, and no sustained oscillatory viral loads will be observed. In contrast to the bifurcation results in the literatures $[4,25,26,30]$, we may be able to rule out the possibility of Hopf bifurcation from the endemic equilibrium as this equilibrium loses its stability. On the other hand, the dynamics of an extended model, containing a class of non-monotone incidence rates (e.g. $\left.f(x, v) v=\frac{x v}{1+\alpha v^{2}}\right)$ incorporating inhibition effect of diseases, is still unclear. Contribution to such open problems including cell-to-cell transmission, cell-mediated immune responses and multi-stage infected progression for activated infected cells (cf. [21, 23]) would also be our future works.

\section{Acknowledgment}

Enatsu is partially supported by JSPS Fellows, No. 257819 and Grant-in-Aid for Young Scientists (B), No. 26800066 of Japan Society for the Promotion of Science. Wang is supported by National Natural Science Foundation of China (Nos. 11401182 and 11471089) and the Science and Technology Innovation Team in Higher Education Institutions of Heilongjiang Province No. 2014TD005. Kuniya is partially supported by Grant-in-Aid for Young Scientists (B), No. 15K17585 of Japan Society for the Promotion of Science.

\section{References}

[1] R. Arnaout, M. Nowak, D. Wodarz, HIV-1 dynamics revisited: Biphasic decay by cytotoxic lymphocyte killing?, Proc. Roy. Soc. Lond. B., 267 (2000), 1347-1354. 1

[2] J. R. Beddington, Mutual interference between parasites or predators and its effect on searching efficiency, J. Animal Ecol., 44 (1975), 331-340. 1

[3] S. Bonhoeffer, J. M. Coffin, M. A. Nowak, Human immunodeficiency virus drug therapy and virus load, J. Virol., 71 (1997), 3275-3278. 1

[4] M. S. Ciupe, B. L. Bivort, D. M. Bortz, P. W. Nelson, Estimating kinetic parameters from HIV primary infection data through the eyes of three different mathematical models, Math. Biosci., 200 (2006), 1-27. 1, 4

[5] D. L. DeAngelis, R. A. Goldstein, R. V. O'Neill, A model for trophic interaction, Ecology, 56 (1975), 881-892. 1

[6] R. J. De Boer, A. S. Perelson, Towards a general function describing T cell proliferation, J. Theoret. Biol., 175 (1995), 567-576. 1

[7] R. J. De Boer, A. S. Perelson, Target cell limited and immune control models of HIV infection: A comparison, J. Theoret. Biol., 190 (1998), 201-214. 1

[8] K. Hattaf, N. Yousfi, A. Tridane, Mathematical analysis of a virus dynamics model with general incidence rate and cure rate, Nonlinear Anal. Real World Appl., 13 (2012), 1866-1872. 1

[9] G. Huang, Y. Takeuchi, W. Ma, Lyapunov functionals for delay differential equations model of viral infections, SIAM Journal on Appl. Math., 70 (2010), 2693-2708. 1, 4

[10] Y. Ji, M. Zheng, Dynamics analysis of a viral infection model with a general standard incidence rate, Abst. Appl. Anal., 2014 (2014), 6 pages. 1

[11] T. Kajiwara, T. Sasaki, Y. Takeuchi, Construction of Lyapunov functionals for delay differential equations in virology and epidemiology, Nonlinear Anal. Real World Appl., 13 (2012), 1802-1826. 1, 1, 4 
[12] A. Korobeinikov, Global properties of basic virus dynamics models, Bull. Math. Biol., 66 (2004), 879-883. 1

[13] Y. Kuang, Delay Differential Equations with Applications in Population Dynamics, Academic Press, Boston, (1993). 2, $3,3,3,3,3$

[14] C. C. McCluskey, Y. Yang, Global stability of a diffusive virus dynamics model with general incidence function and time delay, Nonlinear Anal. Real World Appl., 25 (2015), 64-78. 1

[15] Y. Nakata, Global dynamics of a cell mediated immunity in viral infection models with distributed delays, J. Math. Anal. Appl., 375 (2011), 14-27. 1, 1, 4

[16] M. A. Nowak, C. R. M. Bangham, Population dynamics of immune responses to persistent viruses, Science, 272 (1996), 74-79. 1,1

[17] R. Ouifki, G. Witten, Stability analysis of a model for HIV infection with RTI and three intracellular delays, BioSystems, 95 (2009), 1-6. 1

[18] H. Peng, Z. Guo, Global stability for a viral infection model with saturated incidence rate, Abst. Appl. Anal., 2014 (2014), 9 pages. 1

[19] A. S. Perelson, P. W. Nelson, Mathematical analysis of HIV-1 dynamics in vivo, SIAM Rev., 41 (1999), 3-44. 1

[20] J. Prüss, R. Zacher, R. Schnaubelt, Global asymptotic stability of equilibria in models for virus dynamics, Math. Model. Nat. Phenom., 3 (2008), 126-142. 1

[21] J.-L. Wang, M. Guo, X.-N. Liu, Z. Zhao, Threshold dynamics of HIV-1 virus model with cell-to-cell transmission, cellmediated immune responses and distributed delay, Appl. Math. Comput., 291 (2016), 149-161. 4

[22] T. Wang, Z.-X. Hu., F. Liao, W. Ma, Global stability analysis for delayed virus infection model with general incidence rate and humoral immunity, Math. Comp. Simulation, 89 (2013), 13-22. 1, 1

[23] J.-L. Wang, S.-Q. Liu, The stability analysis of a general viral infection model with distributed delays and multi-staged infected progression, Commun. Nonlinear Sci. Numer. Simul., 20 (2015), 263-272. 4

[24] J.-L. Wang, J.-M. Pang, T. Kuniya, Y. Enatsu, Global threshold dynamics in a five-dimensional virus model with cellmediated, humoral immune responses and distributed delays, Appl. Math. Comput., 241 (2014), 298-316. 1, 1, 2, 2, 4

[25] K. Wang, W. Wang, H. Pang, X.-N. Liu, Complex dynamic behavior in a viral model with delayed immune response, Phys. D, 226 (2007), 197-208. 1, 4

[26] Z.-P. Wang, R. Xu, Stability and Hopf bifurcation in a viral infection model with nonlinear incidence rate and delayed immune response, Commun. Nonlinear Sci. Numer. Simul., 17 (2012), 964-978. 1, 1, 4

[27] S. Wang, D. Zou, Global stability of in-host viral models with humoral immunity and intracellular delays, Appl. Math. Model., 36 (2012), 1313-1322. 1

[28] Y.-C. Yan, W. Wang, Global stability of a five-dimensional model with immune responses and delay, Discrete Contin. Dyn. Syst. Ser. B, 17 (2012), 401-416. 1

[29] Z.-H. Yuan, X.-F. Zou, Global threshold dynamics in an HIV virus model with nonlinear infection rate and distributed invasion and production delays, Math. Biosci. Eng., 10 (2013), 483-498. 1, 1, 4

[30] H. Zhu, Y. Luo, M. Chen, Stability and Hopf bifurcation of a HIV infection model with CTL-response delay, Comput. Math. Appl., 62 (2011), 3091-3102. 1, 4 